\title{
A computational study of functional endoscopic sinus surgery and maxillary sinus drug delivery*
}

\author{
M.R. Wofford', J.S. Kimbell', D.O. Frank-Ito',2, V. Dhandha', K.A. McKinney', \\ G.M. Fleischman', C.S. Ebert, Jr. ', A.M. Zanation', B.A. Senior'
}

Rhinology 53: 41-48, 2015

DOI:10.4193/Rhino13.065

*Received for publication:

May 19, 2013

Accepted: November 11, 2014

' Department of Otolaryngology/Head and Neck Surgery, University of North Carolina, Chapel Hill, NC, USA

2Division of Otolaryngology-Head and Neck Surgery, Department of Surgery, Duke University Medical Center, Durham, NC, USA

\begin{abstract}
Background: Topical medication is increasingly used following functional endoscopic sinus surgery (FESS). Information on particle sizes that maximise maxillary sinus (MS) delivery is conflicting, and the effect of antrostomy size on delivery is unclear. The purpose of this study was to estimate antrostomy and particle size effects on topical MS drug delivery.

Methodology: Sinonasal reconstructions were created from a pre- and a post-FESS CT scan in each of four chronic rhinosinusitis patients. Additional models were created from each post-FESS reconstruction representing four alternative antrostomy sizes. Airflow and particle deposition were simulated in each reconstruction using computational fluid dynamics for nebulised and sprayed delivery.

Results: MS ventilation and drug delivery increased following FESS, the largest virtual antrostomy led to greatest delivery, and MS delivery was sensitive to particle size. Particles within a 5-18 $\mu \mathrm{m}$ and 5-20 $\mu \mathrm{m}$ size range led to peak MS deposition for nebulised and sprayed particles, respectively. Post-FESS increases in drug delivery varied across individuals and within individuals by the type of antrostomy created.
\end{abstract}

Conclusion: Our findings suggest that FESS, particularly with larger antrostomies, improves topical drug delivery, and that certain particle sizes improve this delivery. Further research is needed to contextualise these findings with other post-surgical effects.

Key words: computer simulation, fluid dynamics, nasal sprays, nebulizer, sinusitis

\section{Introduction}

Functional endoscopic sinus surgery (FESS) is commonly performed in chronic rhinosinusitis (CRS) patients who fail medical management ${ }^{(1)}$. Goals of FESS are to improve mucociliary clearance and ventilation by removing obstructive diseased tissue ${ }^{(2)}$, and FESS is thought to improve topical drug delivery ${ }^{(3,4)}$. The ostiomeatal complex (OMC) is an important region in the function of the anterior ethmoid, frontal, and maxillary sinuses. Surgical treatment of this region includes uncinectomy, maxillary antrostomy, and if needed, ethmoidectomy. However, maxillary antrostomy in FESS is not a standardised procedure ${ }^{(5)}$, and may range from a "small hole" middle meatal antrostomy, to extensive enlargement of the ostium along with resection of the posterior half of the inferior turbinate, akin to a medial maxillectomy ${ }^{(6,7)}$.

Topical approaches can provide locally higher drug concentrations while minimising side effects associated with systemic delivery of antimicrobials and corticosteroids ${ }^{(8)}$, and topical steroids are commonly utilised in pre-FESS and post-FESS patients as part of routine medical management ${ }^{(4,9)}$. There is also interest in topical antibiotic delivery to the sinonasal mucosa ${ }^{(10)}$. While a recent position paper states that topical antibiotics cannot be routinely recommended because of a lack of evidence regarding efficacy ${ }^{(3)}$, it is unclear to what extent those results are due to ineffective delivery. 
Topical drugs can be delivered by nasal drops, spray devices, nebulisation, and irrigation. High volume irrigation effectively removes bacteria, biofilms, and inflammatory by-products, and may produce better coverage of the post-operative sinonasal passages than sprays ${ }^{(11,12)}$. However, the role of nasal irrigation in drug delivery is less clear ${ }^{(13)}$. Because the retention of irrigated fluids is less than $5 \%{ }^{(14)}$ and mucosal contact time during irrigation is short, the dose of medication that a patient receives is likely to be larger with more traditional methods such as sprays (4). This study therefore did not include nasal irrigation as an established method of topical nasal drug delivery. Traditional spray devices produce droplets in approximately the 50-100 $\mu \mathrm{m}$ range (13), and deposit predominantly in the anterior nose ${ }^{(15)}$.Despite this limitation, the efficacy of nasal steroid sprays in the treatment of CRS is well established ${ }^{(4)}$. While there are a variety of nebuliser devices utilising mechanisms such as passive diffusion, vortices, or pulsation, their particles are smaller than from spray pumps, within a 1-30 $\mu \mathrm{m}$ size range ${ }^{(13,16)}$. Nebulised particles achieve greater penetration past the nasal valve and distribution throughout the sinonasal cavity than sprayed particles, and this has been attributed to their smaller size and lower velocity ${ }^{(15,17)}$.

Despite common use of various topical medications to treat CRS, drug delivery to the maxillary sinus (MS) is not well understood. Several studies have shown greater drug deposition in post-operative sinuses than pre-operatively ${ }^{(2,18)}$, but results differ on optimal particle sizes for delivery. Experimental studies of nebulized particles have suggested that sizes maximising post-operative sinus deposition are either $<1 \mu \mathrm{m}^{(16)}$ or 3-10 $\mu \mathrm{m}$ (19). One experimental study of sprayed particles determined that $5.6 \mu \mathrm{m}$ resulted in higher delivery to the post-operative paranasal sinuses than $16.4 \mu \mathrm{m}{ }^{(20)}$. However, limitations of these experimental studies, including use of only a few particle sizes and measurement in cadavers ${ }^{(16)}$, molds ${ }^{(19,20)}$, or single individuals ${ }^{(19)}$, makes generalisation difficult.

Computational fluid dynamics (CFD) simulations produce reliable ${ }^{(21,22)}$, easily quantifiable information and have been widely utilised in studying aerosol deposition within the nasal passages (21,23-27). CFD approaches have recently been used to examine

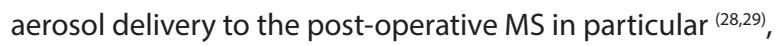
though only in single individuals and the results of Abouali and colleagues ${ }^{(29)}$ were based on virtual modification of a healthy individual.

The goal of this preliminary study was to extend a prior study of maxillary sinus ventilation in pre- and post-surgical states of four CRS patients ${ }^{(30)}$ to investigate the effect of particle size on MS deposition of nebulised and sprayed particles. We sought to 1) determine maximal sinus deposition in each subject pre- and post-operatively and specific particle sizes associated with that deposition, and 2) use virtual surgery models to estimate the effect of a range of possible antrostomies on the magnitude of maximal sinus deposition and the range of particle sizes at which those peaks occur.

\section{Materials and methods}

Patient recruitment and treatment

As described previously ${ }^{(30)}$, four patients with CRS undergoing FESS after failing maximal medical management were recruited to participate in this prospective study, and all provided written, informed consent as required and approved by the Institutional Review Board at the University of North Carolina Hospitals. To ensure a homogenous study population, only patients without polyps and with sinus disease limited to the OMC region with no frontal or sphenoid sinus involvement were included. Subject 1 had bilateral disease and underwent bilateral FESS, while all other subjects had unilateral disease and FESS on that side only. Each subject received a fine-cut $(\leq 0.6 \mathrm{~mm}$ ) pre-FESS CT scan of their paranasal sinuses as part of standard care. Since nasal air-tissue contrast is clearer in CT scans than in MRI scans, and to avoid confounding effects of differing imaging modalities, an additional fine-cut axial CT scan was obtained at least 12 weeks post-FESS in each subject using a cone beam scanner following the "as low as reasonably achievable" protocol.

\section{Sinonasal model construction}

Mimics $^{\mathrm{TM}} 13.1$ (Materialise, Inc., Plymouth, MI, USA) software was used to generate anatomically realistic $3 \mathrm{D}$ reconstructions of the nasal and paranasal sinus airways from the pre- and post-operative CT scans. In each scan, the airspace was selected by setting a maximum threshold of -300 to -351 Hounsfield units based on visual inspection. The resulting pixel selection was then hand-edited to achieve anatomical accuracy. All four subjects received an uncinectomy, maxillary antrostomy, with possible ethmoidectomy, resulting in "standard" maxillary antrostomies of similar sizes $\left(1.08 \text { to } 1.60 \mathrm{~cm}^{2}\right)^{(30)}$. Each post-FESS model was then digitally altered using Mimics ${ }^{\mathrm{TM}}$ to create four other antrostomy types: "large" created by extending the post-surgery antrostomy to the posterior wall of the MS while keeping the standard superior and inferior boundaries; "mega" extended from the large antrostomy by removing the posterior portion of the medial wall of the MS to the floor of the sinus along with resection of the posterior half of the inferior turbinate; and two miniature antrostomies, representing either superior-anterior, "SupAnt," or inferior-posterior,"InfPost," partial uncinectomies using the pre-surgical medial maxillary wall as a guide (Figure 1). These miniature antrostomies were omitted in Subject 4 due to the presence of an accessory ostium.

The 3D reconstructions were exported from Mimics $^{\mathrm{TM}}$ in stereolithography file format and imported into the computeraided design and meshing software ICEM-CFD ${ }^{\text {TM }} 12.1$ (ANSYS, 

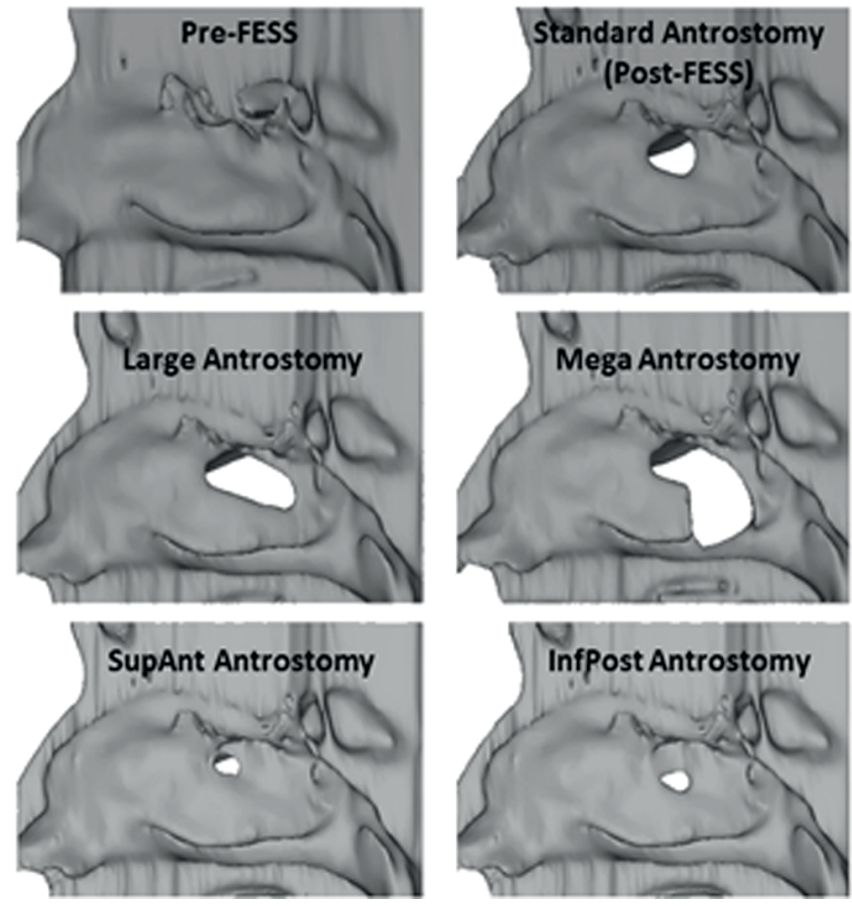

Figure 1. Lateral nasal wall reconstructions showing the opening to the maxillary sinus pre-FESS, post-FESS, and in each type of virtual surgery.

Canonsburg, PA, USA). MS region(s) was marked in each model for regional quantification of particle deposition (Figure 2). A 2-cm outlet tube was added to the nasopharynx to provide fully developed outlet flow for numerical stabilisation, and nostril and outlet planes were defined.

To solve fluid flow and particle trajectory equations, unstructured tetrahedral meshes with prism layers at the air-tissue boundary were created using ICEM-CFD ${ }^{\mathrm{TM}}$. Mesh quality analysis was performed to ensure that distorted (low quality) elements did not impact the accuracy of numerical solutions. Sensitivity analysis on outlet flow and average pressure at the posterior septum showed that the use of a computational mesh consisting of approximately 4 million graded tetrahedral elements resulted in mesh independent airflow results.

\section{Airflow and particle simulations}

Airflow simulations were performed using the CFD software Fluent $^{\mathrm{TM}}$ 12.1.4 (ANSYS, Inc.) under laminar, steady-state flow conditions in the inspiratory direction. Resting flow rates were estimated for each patient by doubling the minute volume predicted by the weight-based curves as described by Garcia et al. (31) and driven by a fixed negative pressure drop from nostrils to outlet. Pressure drops were determined separately for pre- and post-FESS models in each patient, and the post-surgical pressure drop was used for all virtual surgery models. Boundary conditions included setting pressure at the nostrils to zero, setting flow velocity to zero at the tissue boundaries, and assigning driving

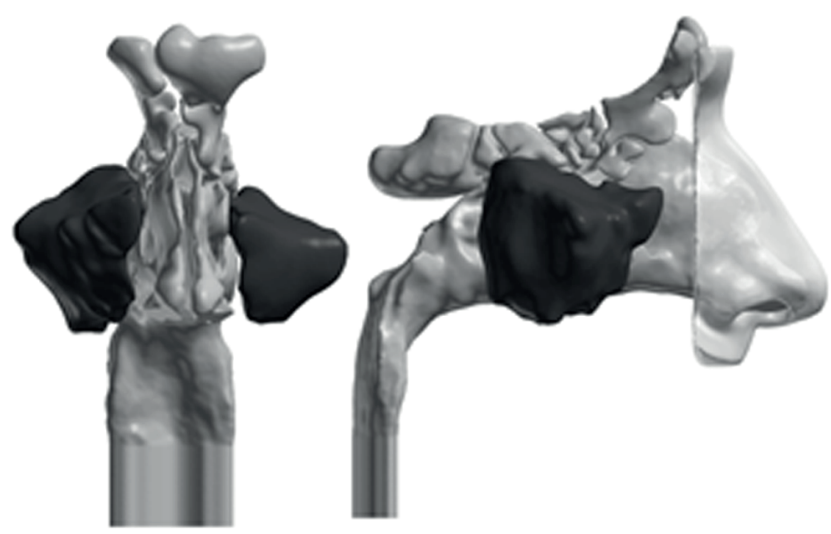

Figure 2. Sinonasal airway reconstruction of Subject 1. Regions shown in dark grey correspond to our definition of the MS region. External nose only included for visualisation.

pressure drops at the outlet, using pressure-inlet, no-slip, and pressure-outlet boundary types, respectively. Airflow allocation to the MS was calculated as the percent of total airflow through the nasal passage on the operated side that circulated through the MS.

Particle trajectories were simulated using the Discrete Phase Model in Fluent ${ }^{\mathrm{TM}}$ with a Lagrangian tracking scheme. Particles were assumed to be spherical and have unit density. To be consistent with prior literature ${ }^{(23,28)}$, particles were assumed to behave like aqueous droplets and expected to adhere to nasal walls on contact. Therefore particles touching the wall were considered trapped, and those reaching the outlet or contacting the tube were considered to have escaped the nasal passages. Particles trapped within the MS were recorded as a fraction of total number of particles released from the nostril on that side. Simulated particle deposition presented in this study was reported only where these particles initially deposited, and no interactions between particles and mucociliary clearance mechanisms were simulated.

To simulate nebulised delivery, particles were released evenly across the nostril plane with no initial velocity. Particle aerodynamic diameters were studied in 1- $\mu$ m increments across a 1-30 $\mu \mathrm{m}$ size range discussed in the literature ${ }^{(13,16)}$. Additional mesh sensitivity analysis conducted on total nasal deposition fraction (DF) indicated that the use of four $0.1 \mathrm{~mm}$-thick prism layers produced mesh-independent particle deposition results. A sensitivity analysis of both MS and total nasal DF to the number of particles released across the nostril plane was performed, indicating that 10,000 particles released per nostril plane was sufficient for results independent of particle number. Using similar parameters as previous studies ${ }^{(24,27,28,32)}$, idealised spray simulations were performed under the same flow 


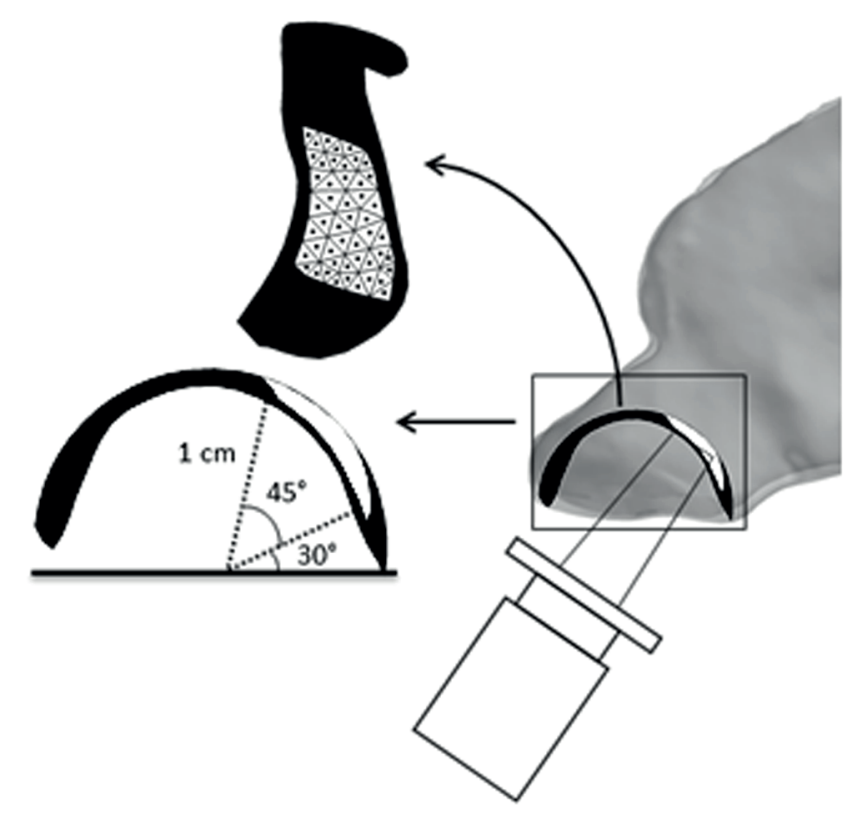

Figure 3. Side view of the anterior nasal airway illustrating our spray setup. The black spherical component represents all regions within the nose at a depth of $1 \mathrm{~cm}$ from the centre of the nostril plane, and the white region is the area of spray release in a sweep from $75^{\circ}$ to $30^{\circ}$ from the horizontal with a 1-mm lateral border. The lattice of release points is shown by the dots within the white region in the $2 \mathrm{D}$ representation of the release surface. Tip of a spray nozzle only included for visualisation. Figure modified from Kimbell et al. ${ }^{(24)}$.

conditions. Particle diameters were studied in $5-\mu \mathrm{m}$ increments from 5-110 $\mu \mathrm{m}$, approximating the range of particle sizes produced by typical spray devices ${ }^{(33)}$, similar to the range used in other CFD studies ${ }^{(27,28,32)}$. The spray cone angle was set to $68^{\circ}$ and 1,000 particles released to represent each cone. An initial velocity of $3 \mathrm{~m} / \mathrm{s}$ was given to sprayed particles, representing the lower end of the $1.5-14.7 \mathrm{~m} / \mathrm{s}$ range found in commercial products ${ }^{(34)}$, as previous studies have indicated poor penetration past the nasal valve at higher velocities ${ }^{(24)}$. Because of random particle distribution within the cones, reported results represent the average of five simulations based on previous work by Frank et al. ${ }^{(27,32)}$. A lattice of approximately 50 spray release points was constructed to represent the physical limits of comfortable nozzle use ${ }^{(24)}$. Release points were equidistant from the centre of the nostril surface at a depth of $1 \mathrm{~cm}$, and placed across a sweep from $75^{\circ}$ to $30^{\circ}$ from the horizontal. A 1-mm gap was left along the lateral wall to account for nozzle width (Figure 3). The direction of each spray was defined from the nostril centre point to each release point. Because Kimbell and colleagues found that penetration of sprayed particles past the nasal valve varied significantly depending on the position and direction of release ${ }^{(24)}$, spray results are reported as an aggregate from all release points. A spray nozzle was not included in the models.

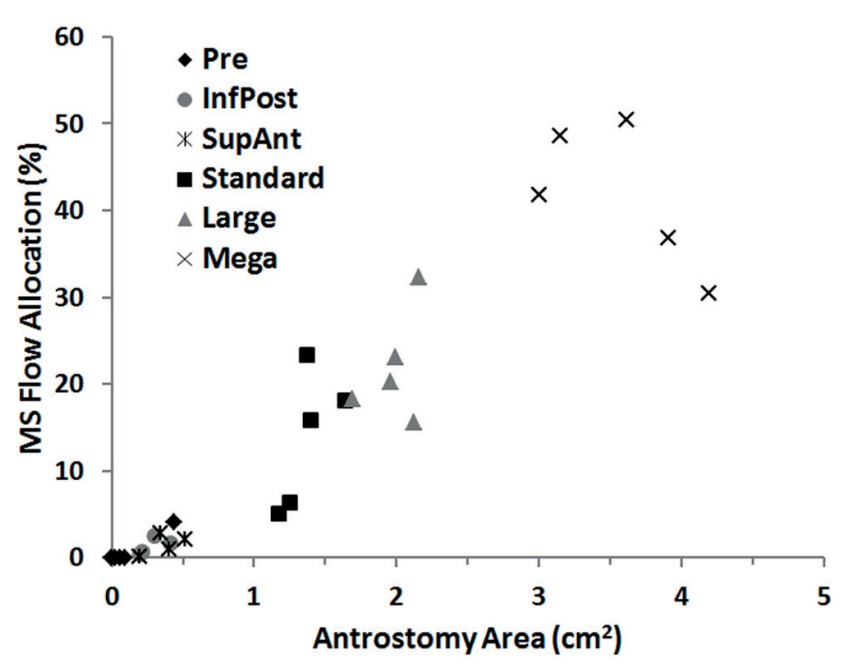

Figure 4. Ostium/antrostomy surface area and airflow allocation to the maxillary sinus for pre-surgery, post-surgery (our standard antrostomy) and each of the virtual surgeries (InfPost, SupAnt, Large, Mega; see text for description) for all four subjects. Maxillary sinus (MS) flow allocation is expressed as a percent of the total airflow through the nasal passage on the operated side.

\section{Results}

\section{MS airflow}

As indicated in our prior report ${ }^{(30)}$, airflow allocation to the MS before surgery was essentially zero, except in the case of Subject 4 who had an accessory ostium. Following surgery and virtual modifications to the maxillary antrostomy, this allocation ranged from $0.2 \%$ to $50.5 \%$ of the total airflow on the side where surgery or virtual alteration was done. In all individuals, each increase in size above a miniature antrostomy always led to increased allocation (Figure 4).

\section{Particle deposition}

Almost no nebulised or sprayed particles deposited in the pre-surgery sinuses. For both modes of delivery, MS DF was less than $0.05 \%$ in all cases except Subject 4 in which there was an accessory ostium. In Subject 4, pre-surgery MS DF peaked at $1.1 \%$ for nebulised particles and $0.3 \%$ for sprays aggregated over all potential release points explored here. Particle sizes that maximised nebulised MS DF ranged from 7 to $16 \mu \mathrm{m}$ among the four subjects in this preliminary study and were similar to those that maximised sprayed MS DF (5-20 $\mu$ m; Figure 5). Peak nebulised MS DF ranged from $0.5 \%$ to $4.9 \%$ and sprayed MS DF ranged from $0.6 \%$ to $2.0 \%$.

In the virtual modifications, particle sizes that maximised MS DF were similar to those for post-surgery, ranging from 5-18 $\mu \mathrm{m}$ and 5-20 $\mu \mathrm{m}$ for nebulised and sprayed delivery, respectively. None of these sizes in the virtual cases differed by more than 4 $\mu \mathrm{m}$ with nebulised particles or $10 \mu \mathrm{m}$ with sprayed from those 


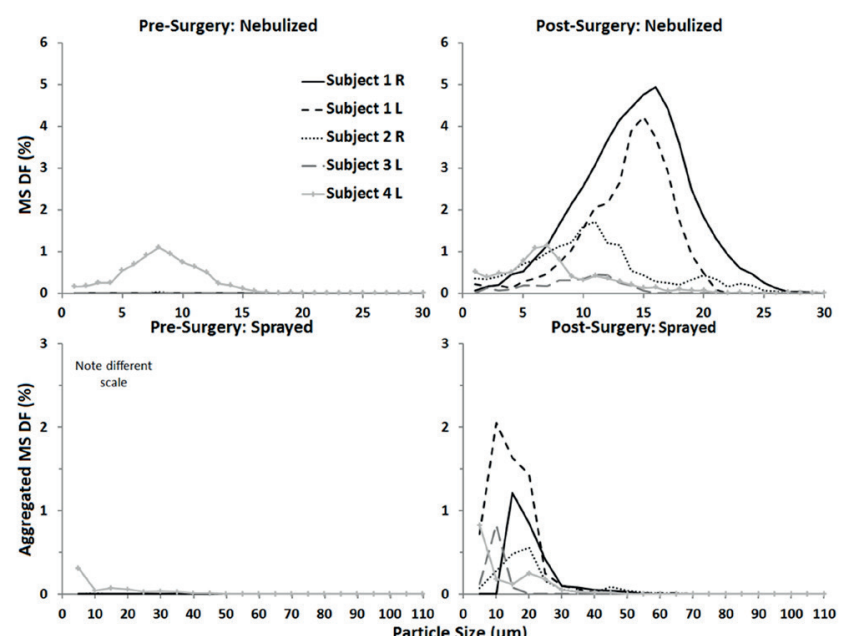

Figure 5. Maxillary sinus deposition fraction pre- and post-surgery as a function of particle size for nebulised and sprayed delivery. Each line corresponds to a given subject. Reported results for sprayed delivery are aggregated from approximately 50 different release points.

that maximised MS DF in the standard antrostomy. The mega antrostomy resulted in the highest nebulised and sprayed MS DF in all subjects, but gains over peak post-surgery MS DF were variable, ranging from 0.4 to 16.7 percentage points for nebulised and from 0.1 to 26.5 percentage points for sprayed particles. The miniature antrostomies consistently resulted in the least deposition of nebulised and sprayed particles among the virtual modifications for all subjects (Figures 6 and 7). While the largest antrostomy consistently resulted in highest peak deposition, increasing antrostomy size did not always lead to increased peak deposition. In 3 of 5 cases, increasing antrostomy size from the standard to large actually decreased peak deposition for both modes of delivery.

\section{Discussion}

\section{MS airflow}

In agreement with previous work, increasing the size of the natural ostium was shown to increase the distribution of airflow into the MS ${ }^{(30,35-37)}$. However, a cadaveric sheep study by Brumund et al. found no further significant increase in MS airflow from increasing antrostomy size from 2-4 $\mathrm{mm}$ to 6-9 $\mathrm{mm}^{(37)}$, and a CFD study by Zang et al. demonstrated little change in MS airflow between antrostomies 8 to $15 \mathrm{~mm}$ in size ${ }^{(38)}$. Our results suggest that larger antrostomies may further increase airflow, resulting in a substantial portion of the total nasal airflow entering the MS. This discrepancy may be because the size of our antrostomies was somewhat greater than in these other studies.

\section{Particle deposition}

Our study builds on existing evidence comparing pre- and postFESS delivery. In a cadaveric study, St Martin et al. found a signi-

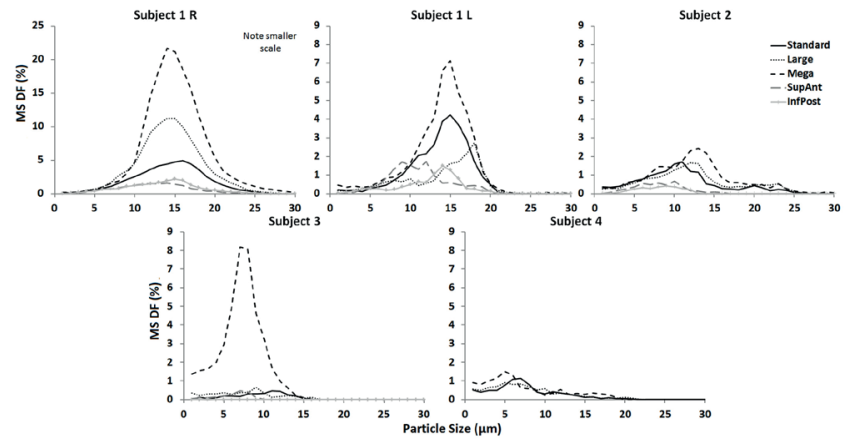

Figure 6. Maxillary sinus deposition fraction as a function of particle size for nebulised delivery. Each line corresponds to a given antrostomy within that subject.

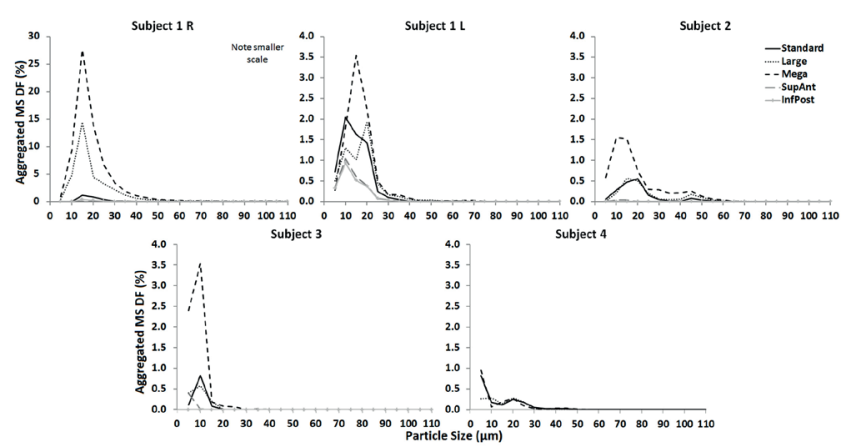

Figure 7. Maxillary sinus deposition fraction as a function of particle size for sprayed delivery. Each line corresponds to a given antrostomy within that subject. Reported results for sprayed delivery are aggregated from approximately 50 different release points.

ficant increase in post-surgical deposition to the MS using particles with a mass mean aerodynamic diameter of $1 \mu \mathrm{m}$, although deposition occurred in both pre- and post-states ${ }^{(18)}$. In another cadaver study, Harvey et al. found that FESS significantly improves delivery to the MS using three different delivery devices ${ }^{(2)}$. Likewise, the CFD study of nebulised delivery by Abouali et al. found that FESS provides an approximately 10-fold increase in drug delivery to the MS ${ }^{(29)}$. Only Hwang et al., in comparing normal subjects and post-FESS patients, found less paranasal sinus delivery in the post-surgical cohort using a vortex nebuliser ${ }^{(39)}$. Our prediction that there is essentially no drug delivery to the MS in the absence of a patent accessory ostium across our entire spectrum of particle sizes is consistent with these reports. The magnitude of delivery post-FESS was generally modest, up to $4.9 \%$ with nebulised and $2.0 \%$ with sprayed, which is comparable to the magnitude of delivery found in other CFD studies ${ }^{(28,29)}$. While we would anticipate that the ethmoid and frontal sinuses would also be more accessible to topical medication post-FESS, we did not attempt to quantify these changes in the present study although similar methods could be applied to do so. It has been established that since smaller particles have less inertia, 
they tend to follow streamlines and largely bypass the nasal passages, whereas larger particles deposit in anterior regions of the nose due to inertial impaction ${ }^{(15)}$. These findings imply that the particle size for maxillary delivery should be small enough to follow streamlines and penetrate past the nasal valve, but also large enough to deposit within the nasal passages. The study by Abouali and colleagues, which involved a virtual uncinectomy and middle meatal antrostomy of a healthy volunteer, investigated a similar method of delivering nebulised microparticles of several sizes and found that peak MS deposition occurred with $10 \mu \mathrm{m}$ particles ${ }^{(29)}$. Likewise, Chen and colleague also found a peak MS deposition with $10 \mu \mathrm{m}$ particles from a simulated sprayed delivery in a single, post-surgical patient ${ }^{(28)}$. Our nebulised size range of 5-16 $\mu \mathrm{m}$ agrees with the above reports suggesting $10 \mu \mathrm{m}$, and is somewhat larger than the approximately 1 and $5.67 \mu \mathrm{m}$ recommended sizes from experimental studies ${ }^{(16,20)}$. This size range was consistent for sprayed particles at 5-20 $\mu \mathrm{m}$, with possible implications for targeting this size range by delivery devices to improve therapeutic drug delivery. MS deposition is highly sensitive to particle size, and the 'ideal' particle size varies both across individuals, and to a lesser degree, by the type of antrostomy created.

Hyo et al. predicted that deposition in the MS is function of the particle size, pressure gradient between the nasal cavity and sinus, and ostium size; with ostium size likely having the dominant effect ${ }^{(19)}$. In agreement with this hypothesis, our study found that the largest virtual antrostomy resulted in the greatest particle deposition. Counter to expectations, however, the large antrostomy resulted in less MS deposition than the standard antrostomy in the majority of nebulised and sprayed cases. In other cases, only a marginal gain was predicted relative to a standard post-FESS by creating a mega antrostomy. These findings suggest competing forces that drive MS delivery between increasing ventilation and increasing antrostomy surface area.

\section{Limitations}

Our study involves a number of simplifications and limitations. Most of the patients in our group had unilateral sinus disease and FESS ( 3 of 4), which may not be generalizable to patients with bilateral FESS. This study is too small to make any statistical inference that these groups may have different outcomes with respect to particle deposition. Particles were assumed to be spherical in shape, and effects of evaporation or particle coagulation were not addressed. Consistent with Hahn and colleagues' findings under moderate flow conditions ${ }^{(41)}$, our study utilised laminar flow conditions. Whether larger antrostomies alter this flow condition has not been investigated. Additionally, this study used steady-state airflow; how maxillary deposition would differ under time-dependent flow is another area for future work.

Our particle transport simulations predicted only initial deposi- tion locations and did not consider effects of mucociliary activity or other "runoff" effects on subsequent particle transport, factors that could affect post-surgical outcome. Similar to other CFD studies, the effects of facial features and spray nozzle on airflow and particle distribution were ignored. However, a recent study by Li et al. demonstrated that inclusion of an external face may alter flow patterns and increase particle deposition ${ }^{(26)}$. Likewise, Inthavong et al. found that a spray nozzle created swirling vortices that increased particle dispersion ${ }^{(25)}$.

Although this study demonstrated that larger antrostomies may optimise topical drug delivery, there are other important considerations in deciding which antrostomy to perform that were not addressed. As the MS is naturally shielded from airflow by the uncinate process, the alterations in airflow to the MS resulting from the larger antrostomies may have significant effects for such issues as increasing mucosal drying or delivery of allergens. Further research is needed to provide context for our findings on drug delivery with these other important considerations before translating this work to clinical practice.

\section{Conclusions}

To the best of our knowledge, this is the largest CFD study of topical drug delivery to the MS to date and presents preliminary quantitative findings on MS delivery of both nebulised and sprayed medication in pre-, post-, and virtual FESS cases. Deposition behaviour was further characterised by the size of the drug particles used in each scenario. Based on the above results of this preliminary study, we conclude that:

1. Our study supports the greater feasibility of topical medical therapy for the post-surgical MS. We have expanded on previous studies by suggesting that larger antrostomies generally increase drug delivery, although there appear to be competing mechanisms for mid-sized antrostomies.

2. Drug delivery to the MS is highly sensitive to particle size. In order for deposition to occur in the post-surgical sinus, appropriately sized particles must be used. Peak delivery occurred with particle sizes within 5-18 $\mu \mathrm{m}$ and 5-20 $\mu \mathrm{m}$ ranges for nebulised and sprayed delivery, respectively. Because of this variability, it may be beneficial to use particles across these size ranges rather than targeting a single size.

3. We saw considerable inter-individual variability in postFESS delivery, and within an individual based on antrostomy size. While it is unknown what constitutes clinically significant delivery, our results suggest that topical medication may not be a viable option in all patients, or with certain antrostomy types in a given individual insofar as delivery to the MS is concerned. By predicting the degree of drug delivery that can be anticipated for a given combination of individual sinonasal anatomy and antrostomy size, use of a CFD environment may have a role in clinical decision making about the use of topical medication post-FESS. 


\section{Acknowledgements}

This work was supported in part by NIH Training Grant T32 DC005460.

\section{Authorship contribution}

Design: MRW, JSK, DOF, VD, CSE, AMZ, BAS. Patient recruitment and treatment: VD, KAM, GMF, CSE, AMZ, BAS. Model construction and acquisition of data: MRW, JSK, DOF, VD, KAM, GMF.
Analysis and interpretation of data: MRW, JSK, DOF, VD, KAM, GMF, CSE, AMZ, BAS. Drafting of the manuscript: MRW. Revision of the manuscript: MRW, JSK, DOF, KAM, GMF, CSE, BAS, CSE. Final approval of manuscript: MRW. JSK, DOF, VD, KAM, GMF, CSE, AMZ, BAS.

\section{Conflict of interest}

There are no conflicts of interest for any of the authors.

\section{References}

1. Dykewicz MS, Hamilos DL. Rhinitis and sinusitis. J Allergy Clin Immunol. 2010; 125 S103-115

2. Harvey RJ, Goddard JC, Wise SK, Schlosser RJ. Effects of endoscopic sinus surgery and delivery device on cadaver sinus irrigation. Otolaryngol Head Neck Surg. 2008; 139 137-142.

3. Fokkens WJ, Lund VJ, Mullol J, et al. European Position Paper on Rhinosinusitis and Nasal Polyps 2012. Rhinology. 2012 Suppl 23: 1-298.

4. Rudmik L, Hoy M, Schlosser RJ, et al. Topical therapies in the management of chronic rhinosinusitis: an evidence-based review with recommendations. Int Forum Allergy Rhinol. 2013: 3: 281-298.

5. Catalano PJ, Strouch M. The minimally invasive sinus technique: theory and practice. Otolaryngol Clin North Am. 2004; 37: 401 409.

6. Cho DY, Hwang PH. Results of endoscopic maxillary mega-antrostomy in recalcitrant maxillary sinusitis. Am J Rhinol. 2008; 22: 658-562.

7. Rodriguez MJ, Sargi Z, Casiano RR. Extended maxillary sinusotomy in isolated refractory maxillary sinus disease. Otolaryngol Head Neck Surg. 2007; 137: 508-510.

8. Rosenfeld RM, Andes D, Bhattacharyya N, et al. Clinical practice guideline: adult sinusitis. Otolaryngol Head Neck Surg. 2007; 137 s1-31.

9. Snidvongs K, Kalish L, Sacks R, Craig JC, Harvey RJ. Topical steroid for chronic rhinosinusitis without polyps. Cochrane Database Syst Rev 2011; 8: CD009274

10. Lim M, Citardi MJ, Leong JL. Topical antimicrobials in the management of chronic rhinosinusitis: a systematic review. Am J Rhinol. 2008; 22: 381-389.

11. Beule A, Athanasiadis T, Athanasiadis E, Field J, Wormald PJ. Efficacy of different techniques of sinonasal irrigation after modified Lothrop procedure. Am J Rhinol Allergy. 2009; 23: 85-90.

12. Miller TR, Muntz HR, Gilbert ME, Orlandi RR Comparison of topical medication delivery systems after sinus surgery. Laryngoscope 2004; 114: 201-204.

13. Albu S. Novel drug-delivery systems for patients with chronic rhinosinusitis. Drug Des Devel Ther. 2012; 6: 125-132.

14. Harvey RJ, Debnath N, Srubiski A, Bleier B Schlosser RJ. Fluid residuals and drug expo- sure in nasal irrigation. Otolaryngol Head Neck Surg. 2009; 141: 757-761.

15. Suman JD, Laube BL, Dalby R. Comparison of nasal deposition and clearance of aerosol generated by nebulizer and an aqueous spray pump. Pharm Res. 1999; 16: 16481652.

16. Hilton C, Wiedmann $T$, St Martin $M$, Humphrey B, Schleiffarth R, Rimell F. Differential deposition of aerosols in the maxillary sinus of human cadavers by particle size. Am J Rhinol. 2008; 22: 395-398.

17. Kundoor V, Dalby RN. Assessment of nasal spray deposition pattern in a silicone human nose model using a color-based method. Pharm Res. 2010; 27: 30-36.

18. St Martin MB, Hitzman CJ, Wiedmann TS, Rimell FL. Deposition of aerosolized particles in the maxillary sinuses before and after endoscopic sinus surgery. Am J Rhinol. 2007; 21: 196-197.

19. Hyo N, Takano H, Hyo Y. Particle deposition efficiency of therapeutic aerosols in the human maxillary sinus. Rhinology. 1989; 27: 17-26.

20. Saijo R, Majima Y, Hyo N, Takano H. Particle deposition of therapeutic aerosols in the nose and paranasal sinuses after transnasal sinus surgery: a cast model study. Am J Rhinol. 2004; 18: 1-7.

21. Farhadi Ghalati P, Keshavarzian E, Abouali O, Faramarzi A, Tu J, Shakibafard A. Numerical analysis of micro- and nano-particle deposition in a realistic human upper airway. Computers in biology and medicine 2012; 42: 39-49

22. 22. Schroeter JD, Tewksbury, E.W., Wong, B.A., Kimbell, J.S. Experimental Measurements and Computational Predictions of Regional Particle Deposition in a Sectional Nasal Model. J Aerosol Med Pulm Drug Deliv. 2014 Feb 28. [Epub ahead of print].

23. Moghadas $H$, Abouali O, Faramarzi A Ahmadi G. Numerical investigation of septal deviation effect on deposition of nano/ microparticles in human nasal passage. Respir Physiol Neurobiol. 2011; 177: 9-18.

24. Kimbell JS, Segal RA, Asgharian B, et al. Characterization of deposition from nasal spray devices using a computational fluid dynamics model of the human nasal passages. J Aerosol Med. 2007; 20: 59-74.

25. Inthavong K, Ge Q, Se CM, Yang W, Tu J. Simulation of sprayed particle deposition in a human nasal cavity including a nasal spray device. J Aerosol Sci 2011; 42: 100113

26. Li $\mathrm{X}$, Inthavong $\mathrm{K}$, Tu J. Particle inhalation and deposition in a human nasal cavity from the external surrounding environment. Build Environ. 2012; 47: 32-39.

27. Frank DO, Kimbell JS, Pawar S, Rhee JS. Effects of anatomy and particle size on nasal sprays and nebulizers. Otolaryngol Head Neck Surg. 2012; 146: 313-319.

28. Chen XB, Lee HP, Chong VF, Wang DY. Drug delivery in the nasal cavity after functional endoscopic sinus surgery: a computational fluid dynamics study. J Laryngol Otol. 2012; 126: 487-494.

29. Abouali O, Keshavarzian E, Farhadi Ghalati $P$ Faramarzi A, Ahmadi G, Bagheri MH. Micro and nanoparticle deposition in human nasal passage pre and post virtual maxillary sinus endoscopic surgery. Respir Physiol Neurobiol. 2012; 181: 335-345.

30. Frank DO, Zanation AM, Dhandha VH, et al. Quantification of airflow into the maxillary sinuses before and after functional endoscopic sinus surgery. Int Forum Allergy Rhinol. 2013; 3: 834-840.

31. Garcia GJ, Schroeter JD, Segal RA, Stanek J, Foureman GL, Kimbell JS. Dosimetry of nasal uptake of water-soluble and reactive gases: a first study of interhuman variability. Inhal Toxicol. 2009; 21: 607-618.

32. Frank DO, Kimbell JS, Cannon D, Pawar SS, Rhee JS. Deviated nasal septum hinders intranasal sprays: a computer simulation study. Rhinology. 2012; 50: 311-318.

33. Cheng YS, Holmes TD, Gao J, et al. Characterization of nasal spray pumps and deposition pattern in a replica of the human nasal airway. J Aerosol Med. 2001; 14: $267-280$

34. Southall J, Newell H, Dickens C, Al-Suleimani Y, Abduljalil H, Yule A. Characterization of particle deposition and penetration from current nasal spray devices. Part I-Laboratory characterisation of nasal spray pumps. J Aerosol Med. 2003; 16: 205

35. Xiong G, Zhan J, Zuo K, Li J, Rong L, Xu $G$. Numerical flow simulation in the postendoscopic sinus surgery nasal cavity. Med Biol Eng Comput. 2008; 46: 1161-1167.

36. Hood CM, Schroter RC, Doorly DJ, Blenke EJ, Tolley NS. Computational modeling of flow and gas exchange in models of the human maxillary sinus. J Appl Physiol. 2009; 107: 1195-1203. 
37. Brumund KT, Graham SM, Beck KC, Hoffman EA, McLennan G. The effect of maxillary sinus antrostomy size on xenon ventilation in the sheep model.Otolaryngol Head Neck Surg. 2004; 131: 528-533.

38. Zang H, Liu Y, Han D, et al. Airflow and temperature distribution inside the maxillary sinus: a computational fluid dynamics simulation. Acta Oto-laryngolog. 2012; 132: 637644.

39. Hwang PH, Woo RJ, Fong KJ. Intranasal deposition of nebulized saline: a radionuclide distribution study. AmJ Rhinol. 2006; 20: 255-261.

40. Garcia GJ, Tewksbury EW, Wong BA, Kimbell JS. Interindividual variability in nasal filtration as a function of nasal cavity geometry. J Aerosol Med Pulm Drug Deliv. 2009; 22:
139-155.

41. Hahn I, Scherer PW, Mozell MM. Velocity profiles measured for airflow through a large-scale model of the human nasal cavity. J Appl Physiol. 1993; 75: 2273-2287.
Julia S. Kimbell

Department of Otolarynogology/

Head \& Neck Surgery

University of North Carolina School

of Medicine

170 Manning Dr., CB \#7070

Chapel Hill, NC 27599-7070

USA

Tel: +1 (919) 843-8417

Fax: +1 (919) 966-7941

E-mail:julia_kimbell@med.unc.edu 